\title{
Vibration based condition monitoring of wind turbine gearboxes based on cyclostationary analysis
}

\author{
Alexandre Mauricio, Junyu Qi, Konstantinos Gryllias* \\ Division PMA, Department of Mechanical Engineering \\ Faculty of Engineering Science, KU Leuven \\ Dynamics of Mechanical and Mechatronics Systems, Flanders Make \\ Celestijnenlaan 300, BOX 2420, 3001 Leuven, Belgium \\ Email: konstantinos.gryllias@kuleuven.be
}

\begin{abstract}
Wind industry experiences a tremendous growth during the last few decades. As of the end of 2016, the worldwide total installed electricity generation capacity from wind power amounted to 486,790 MW, presenting an increase of $12.5 \%$ compared to the previous year. Nowadays wind turbine manufacturers tend to adopt new business models proposing total health monitoring services and solutions, using regular inspections or even embedding sensors and health monitoring systems within each unit. Regularly planned or permanent monitoring ensures a continuous power generation and reduce maintenance costs, prompting specific actions when necessary. The core of wind turbine drivetrain is usually a complicated planetary gearbox. One of the main gearbox components which are commonly responsible for the machinery breakdowns are rolling element bearings. The failure signs of an early bearing damage are usually weak compared to other sources of excitation (e.g. gears). Focusing towards the accurate and early bearing fault detection, a plethora of signal processing methods have been proposed including spectral analysis, synchronous averaging and enveloping. Envelope analysis is based on the extraction of the envelope of the signal, after filtering around a frequency band excited by impacts due to the bearing faults. Kurtogram has been proposed and widely used as an automatic methodology for the selection of the filtering band, being on the other hand sensible in outliers. Recently an emerging interest has been focused on modelling rotating machinery signals as cyclostationary, which is a particular class of non-stationary stochastic processes. Cyclic Spectral Correlation and Cyclic Spectral Coherence have been presented as powerful tools for condition monitoring of rolling element bearings, exploiting their cyclostationary behaviour. In this work a new diagnostic tool is introduced based on the integration of the Cyclic Spectral Coherence along a frequency band that contains the diagnostic information. A special procedure is proposed in order to automatically select the filtering band, maximizing the corresponding fault indicators. The effectiveness of the methodology is validated using the National Renewable Energy Laboratory (NREL) wind turbine gearbox vibration condition monitoring benchmarking dataset which includes various faults with different levels of diagnostic complexity.
\end{abstract}
Nomenclature
$\alpha$ Cyclic frequency
$\tau$ Time lag
bw Bandwidth
cf Center frequency
$f$ Spectral frequency
$R_{x}$ Autocorrelation function
$t$ Time
$\mathbb{E}$ Ensemble expected value

*Address all correspondence to this author. 
BPFO Ball Pass Frequency Outer Race

CSC $\gamma_{x}(\alpha, f)$ Cyclic Spectral Coherence

CSCor $S_{x}(\alpha, f)$ Cyclic Spectral Correlation

EES Enhanced Envelope Spectrum

EESFO Enhanced Envelope Spectrum by Feature Optimization

FTF Fundamental Train Frequency

GMF Gear Mesh Frequency

HSS High Speed Shaft

IMS Intermediate Speed Shaft

NREL National Renewable Energy Laboratory

SES Squared Envelope Spectrum

PLC Planet Carrier

\section{INTRODUCTION}

Power generation, from wind turbines and particularly from the offshore ones, has seen a significant drop in bid prices over the past years emerging as a clear trend. In Europe, the cost of 200\$/megawatt-hour estimated for new projects built for commercial use in the period 2017 to 2019 is expected to be dropped to 60\$/megawatt-hour while the United States commissioned its first commercial offshore wind project, which is expected to provide electricity power for 17,000 Rhode Islands homes [1]. Furthermore in September 2017, onshore wind energy appeared to be cheaper than electricity from any other source in UK for the first time [2]. Wind energy is an emerging market for power generation but it should become more cost effective in order to increase its competitiveness in addition to the advantage of clean energy production by extending the turbine lifetime [3]. Onshore and offshore wind turbines are often built in locations with difficult or rather costly access. As a result, the reduction of maintenance cost by monitoring the condition of the wind turbine, in order to avoid unexpected breakdowns [4], appears as one of the main strategies. More specifically, monitoring temperature, vibration and acoustic signals using advanced signal processing tools and diagnostic indicators, allows for accurate and early detection of damage in wind turbines avoiding a catastrophic accident.

A number of advanced signal processing tools have been proposed during the last decades, focusing towards the condition monitoring of components of rotating machinery, such as the bearings and gears of wind turbine epicyclic gearboxes. A classical approach for bearing fault detection is the Envelope Analysis. The raw vibration signal is demodulated (using often the Hilbert Transform in order to calculate the analytical signal) and the analysis of the Squared Envelope Spectrum reveals the harmonics of the characteristic bearing fault frequency (i.e BPFO, BPFI etc). Usually the raw signal is first filtered around an excited frequency band where the SNR is high. The parameters of the filter were initially selected by engineering experience but recently a number of automated methodologies have been proposed by optimising a parameter such as the Spectral Kurtosis [5] (which leads to Kurtogram) and the Peak Energy Criterion [6]. In the case of gearboxes the strong contribution of gears to the measured signals usually masks the rather weak bearing signals. In order to overcome this problem Cepstrum Editing Procedure (CEP) [7] and Cepstrum pre-whitening [8] have been proposed to separate the strong periodic part related to gears and the residual one related to bearings. The former method is able to remove certain harmonics and their sidebands by editing them in the cepstrum domain while the later one present a lower computational cost. Gear and bearing signals are very well described by the theory of cyclostationarity. Signals resulting from phenomena of deterministic nature, such as unbalances, eccentricities, meshing gear faults, etc are cyclostationary signals of order 1 while signals resulting from phenomena of random nature, such as impacts, frictions etc. are of order 2. Antoni [9] exploiting the property of cyclostationarity proposed the use of Cyclic Spectral Coherence (CSC) for the spectral analysis of cyclostationary signals and introduced a new mathematical way of estimating it, focusing to the decrease of the computational time [10]. The application of Cyclic Spectral Coherence leads to a 2D spectral frequency- cyclic frequency map which is often confusing for a non experienced user. For this reason, integrating over the spectral frequency lines has been proposed leading to the Enhanced Envelope Spectrum, which includes all the diagnostic information.

The National Renewable Energy Laboratory (NREL) performed a round robin condition monitoring experiment on a wind turbine gearbox in order to test the capabilities of vibration analysis [11]. Various industrial and academic partners participated to the blind analysis and the findings concluded that a condition monitoring based on vibration analysis approach could successfully diagnose various faults [12]. A methodology based on the application of the high frequency demodulation, the time-synchronous averaging and the correct feature selection led to the detection of faults on three different bearings of the NREL wind turbine gearbox. However, in the blind analysis of the round robin, one of the existing faults, located at the low speed shaft downwind bearing could not be detected [13].

The aim of this paper is the introduction of a new automated methodology for the extraction of a filtered version of the Enhanced Envelope Spectrum which includes, enhances and presents the diagnostic information in a clear way. The methodology is applied and further validated on a NREL wind turbine gearbox dataset, focusing on the diagnostics of wind

GTP-18-1329 - Gryllias - Page 2 
Copyright furbine rolling element bearings. The structure of this paper is as follows. First the theory of the Cyclic Spectral Analysis is briefly presented and the new methodology is introduced. Moreover the NREL wind turbine gearbox test set up is presented. Furthermore the methodology is applied and the results are presented and analysed focusing on bearing diagnostics. Finally the paper closes with some conclusions.

\section{CYCLIC SPECTRAL ANALYSIS}

Fault detection and diagnosis of rolling element bearings is a field of intensive research since they are critical components of rotating machinery. Bearing and gear signals can be modelled as cyclostationary signals. Cyclostationary signals, although they are not necessarily periodic, are produced by a hidden periodic mechanism. The category of cyclostationarity includes stationary signals (assuming an infinite period), non-stationary signals which exhibit periodicity after passing through a non-linear transform and periodic signals as a special case, describing practically a series of mechanical signals generated by rotating and reciprocating machines. A signal is characterised as cyclostationary of order $\mathrm{n}$ when its nth order statistics are periodic. A first-order cyclostationary signal presents a steady first-order statistics, i.e. the mean value in the ensemble average sense is unaltered [9], [10].

$$
\mathbb{E}\{x\}=\mathbb{E}\{p+n\}=p
$$

Similarly, a second-order cyclostationary signal has an autocorrelation function which is a periodic function of time:

$$
R_{x x}(t, \tau)=\mathbb{E}\{x(t-\tau / 2) x(t+\tau / 2)\}=R_{x x}(t+T, \tau)
$$

\section{Cyclic Spectral Coherence}

When available for all values of $\mathrm{t}$ and $\tau$, the autocorrelation function presented in Eq. 2 contains all the information about a second-order cyclostationary signal and the representation in the frequency domain usually provides more insight into the structure of the signal. Since the autocorrelation function is a function of two variables, a two-dimensional Fourier transform is performed, giving rise to the so-called "spectral correlation":

$$
S_{x x}(\alpha, f)=\lim _{W \rightarrow \infty} \frac{1}{W} \int_{\mathbb{R}} \int_{-W / 2}^{W / 2} R_{x x}(t, \tau) e^{-j 2 \pi(f \tau+\alpha t)} d t d \tau
$$

This involves two frequency variables with very different physical meanings. Frequency $f$, being the dual of time-lag $\tau$, indicates the frequency of the carrier signal. Frequency $\alpha$, being the dual of time $t$, indicates the frequency of the modulation and accordingly is usually named the "cyclic frequency" or the "modulation frequency". Hence, the spectral correlation may be interpreted as giving the strength of the elementary waves in signal $\mathrm{x}$ carried and modulated at all possible combinations $(\alpha, f)$. The autocorrelation function of a second-order cyclostationary signal is transient in time-lag $t$ and periodic in time $t$ and as a result the corresponding spectral correlation is continuous in $f$ but discrete in $\alpha$, returning a very distinctive signature. Alternatively the spectral correlation can be calculated as:

$$
S_{x x}(\alpha, f)=\lim _{T \rightarrow \infty} \mathbb{E}\left\{X_{T}(f+\alpha / 2) X_{T}^{*}(f-\alpha / 2)\right\}
$$

where $X_{T}(f)$ stands for the Fourier transform of signal $x(t)$ over an interval of duration T. The 2D plan of cyclic spectral correlation is a powerful tool containing all the available diagnostic information but often an expert is needed to interpret it. On the other hand, the integral of the spectral correlation over all frequency $f$ is the Fourier transform of the expected value of the squared signal and as a result it corresponds to the Spectrum of the Squared Envelope of the signal:

$$
\begin{array}{r}
\int S_{x x}(\alpha, f) d f=\iint \mathbb{E}\left\{x(t+\tau / 2) x^{*}(t-\tau / 2)\right\}\left(\int e^{-2 j \pi f t} d f\right) e^{-j 2 \pi \alpha t} d t d \tau= \\
\iint \mathbb{E}\left\{x(t+\tau / 2) x^{*}(t-\tau / 2)\right\} \delta(\tau) d \tau e^{-j 2 \pi \alpha t} d t=\int \mathbb{E}\left\{x(t) x^{*}(t)\right\} e^{-j 2 \pi \alpha t} d t=\mathcal{F}\left\{\mathbb{E}\left\{|x(t)|^{2}\right\}\right\}
\end{array}
$$

Moreover the Cyclic Spectral Coherence can be estimated as a normalized version of the Cyclic Spectral Correlation with magnitude normalized within 0 and 1 :

$$
\gamma_{x x}(\alpha, f)=\frac{S_{x x}(\alpha, f)}{\sqrt{S_{x x}(0, f) S_{x x}(0, f-\alpha)}}
$$


Copyright fhe Cyclic Spectral Coherence can be interpreted as the Spectral Correlation of a whitened signal, which tends to equalize regions with very different energy levels, magnifying weak cyclostationary signals.

\section{Enhanced Envelope Spectrum by Feature Optimization}

The 2D CSC and CSCor maps include all the diagnostic information but are quite complicate for a non experienced user. As a result the integration over the full band of the spectral frequency lines $\left[f_{1} ; f_{2}\right]$ has been proposed leading to an one-dimensional representation entitled the "Enhanced Envelope Spectrum", which is equivalent to the Squared Envelope Spectrum (SES) and is calculated using the equation 7.

$$
S_{x x}^{E E S}(\alpha)=\int_{f_{1}}^{f_{2}}\left|\gamma_{x x}(\alpha, f)\right| d f
$$

where $f_{1}=0$ and $f_{2}=f_{s} / 2$, equal to the Nyquist frequency. The integral in equation 7 is replaced for numerical implementation by discrete sums over the spectral frequency $f$. As it can be concluded by the equations, the EES $\left(S_{x x}^{E E S}(\alpha)\right)$ demonstrates the modulation (bearing) frequencies and their harmonics present in the processed signals providing a clearer fault detection compared to the classical SES. The EES does not necessarily need to be calculated based on an integration over the full available spectral frequency $f$. Contrary, a specific band of integration between $f_{1}>0$ and $f_{2}<f_{s} / 2$ can be selected resulting to a filtered version of EES in direct equivalence with a band pass filter. The selection of an optimum frequency integration band $\left[f_{1}: f_{2}\right]$ will improve the SNR leading to a clearer envelope spectrum with enhanced harmonics of the characteristic defect frequency. The optimum frequency band should be estimated by the optimisation of a criterion which is related to the quality of the envelope spectrum. Ideally the envelope spectrum of a defective bearing signal should consist mainly of harmonics of the characteristic defect frequency presenting a high amplitude. Based on this observation, the maximization of the indicator $I C$ is proposed in this paper. The indicator $I C$ is described as:

$$
I C=\left.\sum_{n=1}^{N} S_{x x}^{E E S}(\alpha)\right|_{\alpha=n x f_{a}}
$$

where $f_{\alpha}$ is the cyclic frequency (pre-set equal to an expected fault frequency) and $N$ is the number of fault frequency harmonics. It should be noted that unlike with gears or other deterministic modulating functions, the presence of random slip in bearings means that the fault frequency is not known exactly. As a result a tolerance around $\alpha$ should be allowed in the calculation of $I C$. The calculation in this work is restricted to the first three harmonics of the characteristic defect frequencies, i.e. $\mathrm{N}=3$ in equation 8 while the characteristic defect frequency is equal to the Ball Pass Frequency of the Outer Race (BPFO) or of the Inner Race (BPFI). The steps of the general methodology entitled Enhanced Envelope Spectrum by Feature Optimization (EESFO) can be summarised as follows. Initially the 2D CSC map $\left(\gamma_{x x}(\alpha, f)\right)$ is calculated. Moreover different integration bands are considered having as parameters the center frequency and the bandwidth. Each integration $\left[f_{1}: f_{2}\right]$ leads to an EES and the indicator $I C$ is estimated. The optimum band is selected by maximizing the indicator $I C$. In this paper in order to present a simplified version, the bandwidth is selected a priori and it is shifted over the spectral frequency axis as demonstrated in figure 1 . The indicator $I C$ is presented as a function of the central frequency $c f$.

\section{OVERVIEW OF THE WIND TURBINE GEARBOX}

In order to evaluate its effectiveness for accurate and early bearing fault detection and diagnosis, the proposed methodology is tested and evaluated on a publicly available wind turbine gearbox vibration condition monitoring benchmarking dataset provided by the NREL, which investigated the root causes and loading conditions that result in the premature failure of wind turbine gearboxes through a consortium entitled the Gearbox Reliability Collaborative (GRC). In the frames of this initiative, NREL performed a benchmarking data campaign using the NREL Dynamometer Test Facility (DTF). The test turbine is a stall-controlled three bladed upwind turbine with a rated power of $750 \mathrm{~kW}$. The turbine operates at $1800 \mathrm{rpm}$. The complete nacelle and drivetrain was installed in the NREL DTF and was hard fixed to the floor without the hub, rotor, yaw bearing or yaw drives, as presented in figure 2. The actual field controller was used to provide start-up and system safety responses. Two gearboxes, one "healthy" and one "damaged" of the same design have been tested under the GRC dynamometer and vibration data have been collected by accelerometers along with high-speed shaft rpm signals. The "healthy" gearbox was only tested at the dynamometer. The "damaged" gearbox first was run in the dynamometer and was later moved to a wind farm close to NREL for field testing. In the field test, the gearbox experienced two loss-of-oil events that damaged its internal bearings and gear elements. The gearbox was brought back to NREL and condition monitoring equipment was installed and then retested under controlled loading conditions that would not cause catastrophic failure of

GTP-18-1329 - Gryllias - Page 4 


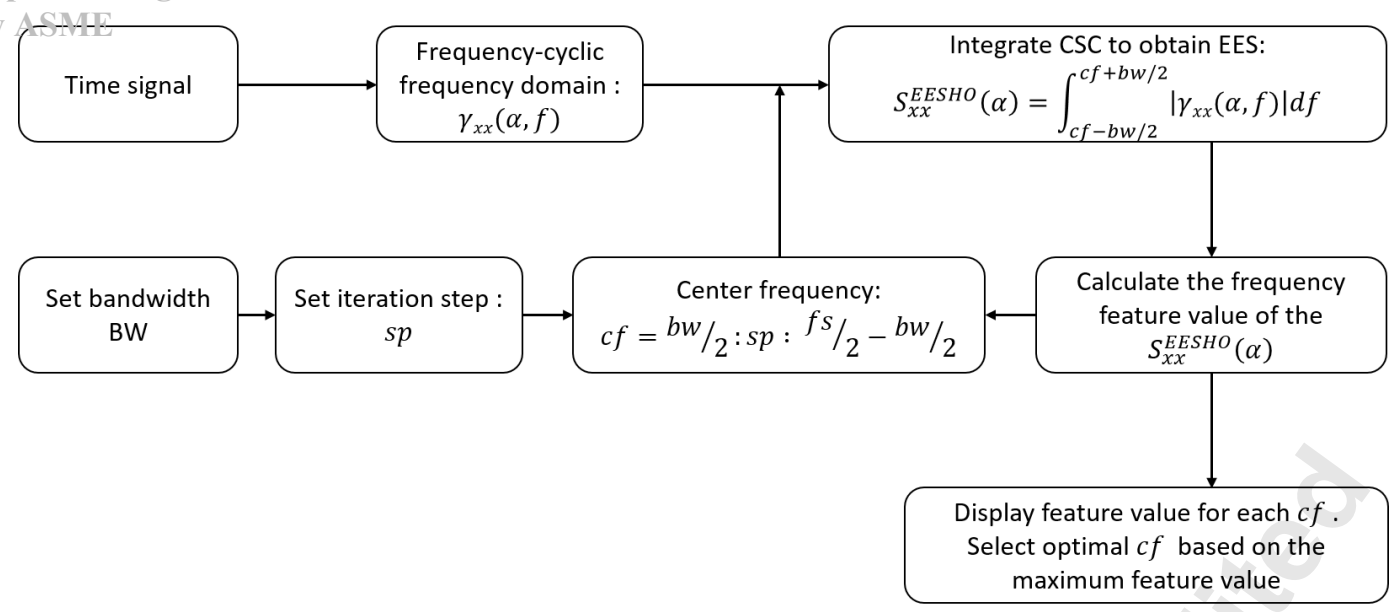

Fig. 1: FLOWCHART OF ENHANCED ENVELOPE SPECTRUM BY FEATURE OPTIMIZATION (EESFO).

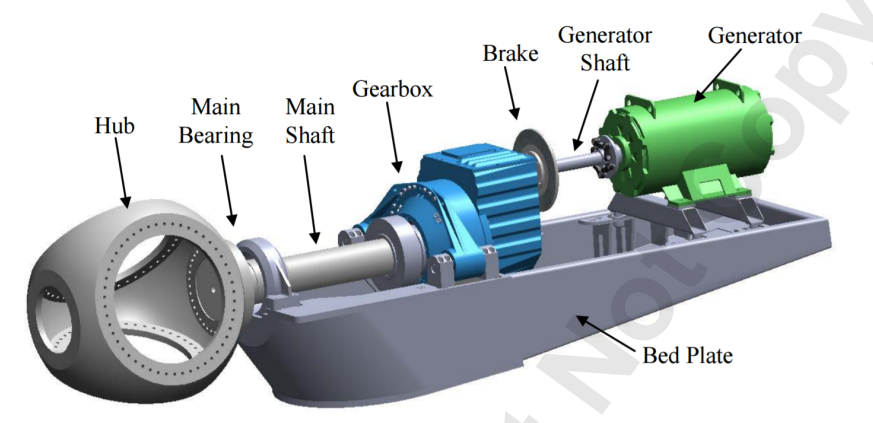

Fig. 2: REPRESENTATION OF THE WIND TURBINE COMPONENTS AND ITS MEASURED GEARBOX [12].

the gearbox. The two test gearboxes were originally taken from the field and redesigned with the specific configuration, rebuilt and instrumented. Both gearboxes have an overall ratio of 1:81:49. Each gearbox is composed of one low speed (LS) planetary stage and two parallel stages. The nomenclature and the bearing location of the test gearboxes is presented in figure 3. Eight accelerometers (AN3 - AN10) mounted on the casing of the gearbox have been used to collect data with a sampling frequency equal to $40 \mathrm{kHz}$ per channel. The sensors AN3 and AN4 are mounted onto the ring gear, close to the lower speed gearbox components. The sensor AN5 is located near to the LSS, the AN6 to the IMS and finally the AN7, AN8 and AN9 to the HSS. Since this paper focuses on bearing faults, the gear faults were not considered. The rotating speed of the different components are presented in orders normalised to the speed of HSS at table 1. Furthermore the various bearing faults and their characteristic defect frequencies are presented in table 2.

\section{APPLICATION OF THE METHODOLOGY AND DISCUSSION}

The methodology presented above has been applied on the signals of the NREL dataset. First of all it is worth mentioning that the defected bearings are better detected by analysing the signals captured by the sensors which are closer to them, i.e. the HSS downwind bearing defects are better detected at the vibration signal captured by the sensors AN7. Initially the signal captured by the sensor AN7 is analysed for both the healthy and the faulty case. The CSC map is estimated and the BPFI harmonics of the HSS downwind bearing are clearly detected at the faulty case. A zoom of the CSC map around the first $(345.3 \mathrm{~Hz})$ and the third harmonic of the BPFI are presented in Fig. 4. The modulation is clear identified at a frequency band between 10 and $15 \mathrm{kHz}$. An integration of the CSC map at this frequency band is expected to provide an enhanced envelope spectrum and an easier detection. The optimisation procedure is applied on the healthy and the damaged case and the indicator values in function of the central frequency of the band are presented in Fig. 5 (b). As expected the maximisation of the indicator at the damaged case leads to the selection of a frequency band around the central frequency $c f=13.1 \mathrm{kHz}$. In the healthy case, the criterion returns a different frequency band located at low frequencies, where the harmonics of the deterministic components related to the gears and shafts are always present and are dominant in the absence of fault. The selected frequency band for the health case, with a central frequency equal to $1.1 \mathrm{kHz}$, is presented in Fig. 5 (a). Based on the selected frequency bands, an integration over the spectral frequency on the CSC map is performed for both the healthy and 


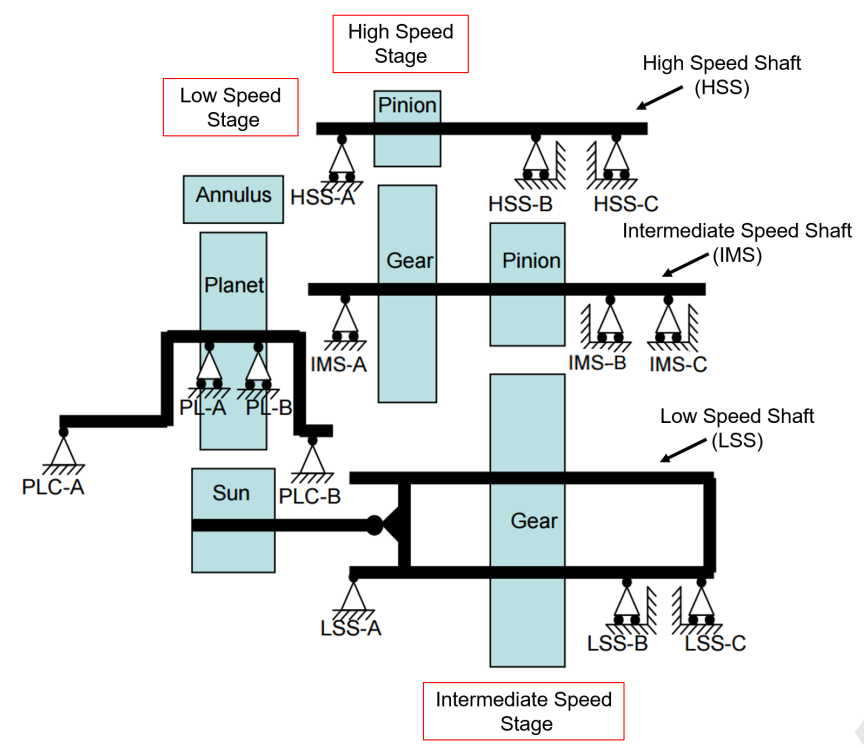

Fig. 3: PLANETARY GEARBOX REPRESENTATION OF BEARING LOCATION AND GEAR STAGES [12].

Table 1: SHAFTS AND GEARS FREQUENCIES.

\begin{tabular}{lcr}
\hline & Order $(/ \mathrm{HSS})$ & Frequency $(\mathrm{Hz})$ \\
\hline Rotor & 0.012 & 0.37 \\
Planets & 0.043 & 1.30 \\
Sun shaft & 0.070 & 2.10 \\
Intermediate Shaft & 0.250 & 7.50 \\
Generator Shaft (HSS) & 1.000 & 30.00 \\
(GMF) Sun $\rightarrow$ IMS & 5.750 & 172.00 \\
(GMF) IMS $\rightarrow$ HSS & 22.000 & 660.00 \\
\hline
\end{tabular}

GMF: Gear Mesh Frequency, HSS: High Speed Shaft, IMS: Intermediate Speed Shaft.

Table 2: CHARACTERISTIC BEARING FAULT FREQUENCIES.

\begin{tabular}{lcrr}
\hline Bearing label & Fault type & Frequency $(\mathrm{Hz})$ & Sensor \\
\hline HSS Downwind & BPFI & 345.3 & AN7 \\
HSS Downwind & FTF & 12.8 & AN7 \\
IMS Downwind & BPFO & 105.3 & AN6 \\
IMS Upwind & BPFI & 73.7 & AN6 \\
PLC Upwind & BPFO & 8.8 & AN5 \\
\hline
\end{tabular}

HSS: High Speed Shaft, IMS: Intermediate Speed Shaft, PLC: Planet Carrier, BPFI: Ball Pass Frequency Inner race, BPFO: Ball Pass Frequency Outer race, FTF: Fundamental Train Frequency.

the damaged case leading to the corresponding Enhanced Envelope Spectra, presented in Fig. 6. The true BPFI is identified in the spectrum of the faulty case equal to $345.3 \mathrm{~Hz}$ and the second and the third harmonics are clearly detected as well. It 


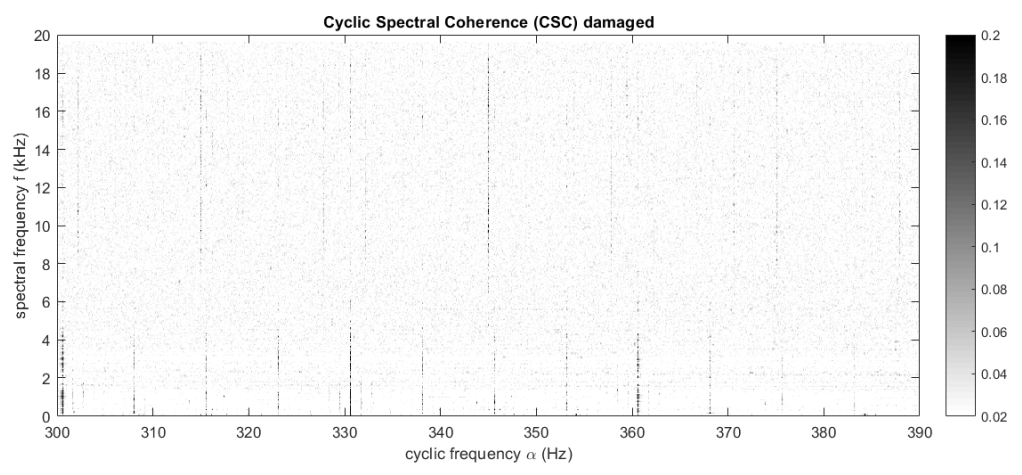

(a)

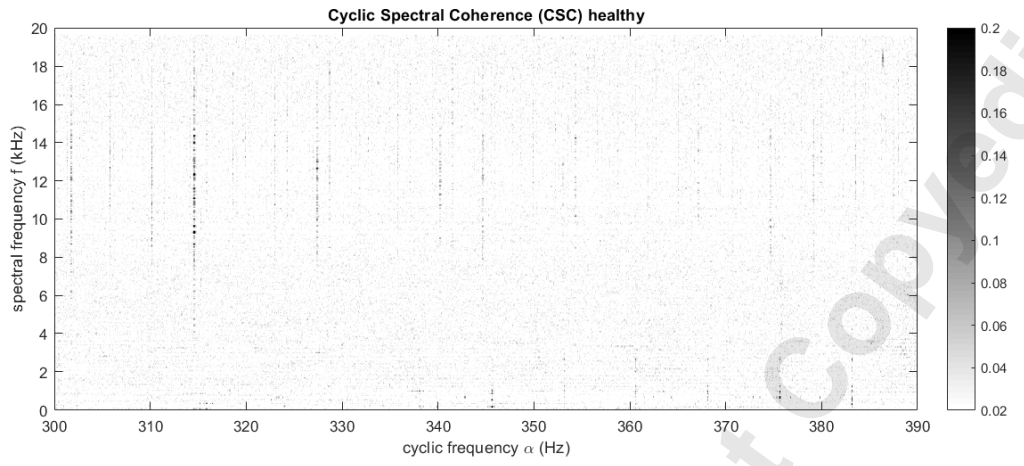

(b)

Fig. 4: CYCLIC SPECTRAL COHERENCE MAP OF SENSOR AN7 ZOOM AROUND THE BPFI FOR: (A) DAMAGED CASE, (B) HEALTHY CASE.

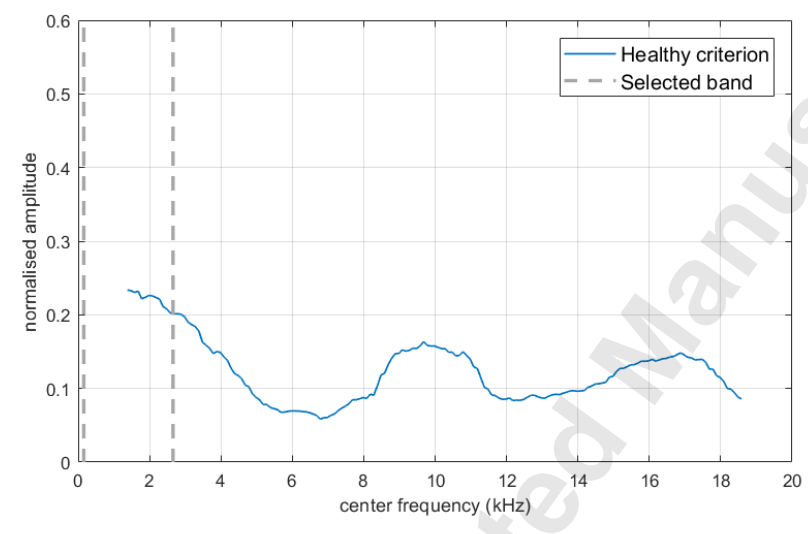

(a)

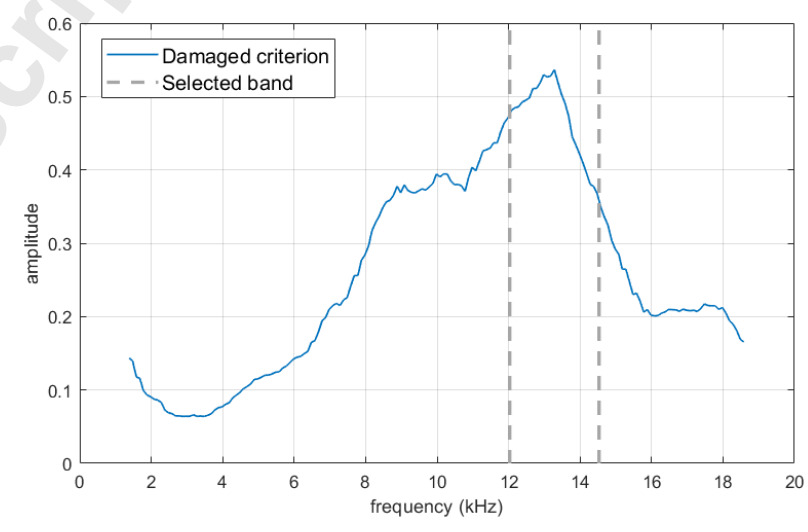

(b)

Fig. 5: EESFO CRITERION FOR THE SELECTION OF BAND OF INTEGRATION OF CSC FOR HSS DOWNWIND BEARING BPFI (345.3 HZ) OPTIMIZATION FOR SENSOR AN7 FOR (A) HEALTHY AND (B) DAMAGED.

should be noted that at the healthy case a peak close to the $2^{\text {nd }}$ harmonic of the BPFI (equal to $690 \mathrm{~Hz}$ ) is identified. However this peak corresponds to a sideband of the Gear Mesh Frequency (GMF equal to $660 \mathrm{~Hz}$ ) modulated by the HSS frequency of $30 \mathrm{~Hz}$. A number of sidebands are clearly identified in the healthy case. A zoom of the Enhanced Envelope Spectrum is presented in Fig. 7 where the classical pattern of BPFI modulated by the HSS is clearly identified. A second modulation due to the Fundamental Train Frequency (FTF) of the HSS downwind bearing can be also detected, which suggests that the cage of the bearing is also damaged. This result is confirmed by the NREL report [12]. The CSC display is not part of the procedure, as it adds complexity to the analysis. It has been presented for the first damage case solely to provide a base understanding and validation of the procedure. In fact, the EESFO method has been developed exactly to avoid the part of the analysis related to the CSC, effectively skipping it in an automatized manner obtaining an Enhanced Envelope Spectrum. 


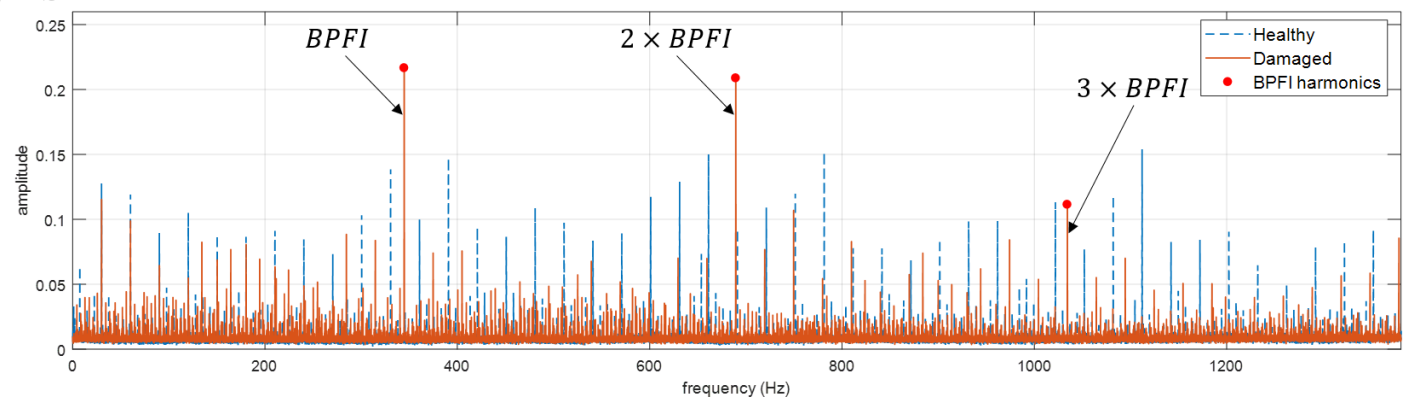

Fig. 6: EESFO FOR THE BPFI DETECTION OF THE HSS DOWNWIND BEARING DAMAGE CORRESPONDING TO SENSOR AN7.

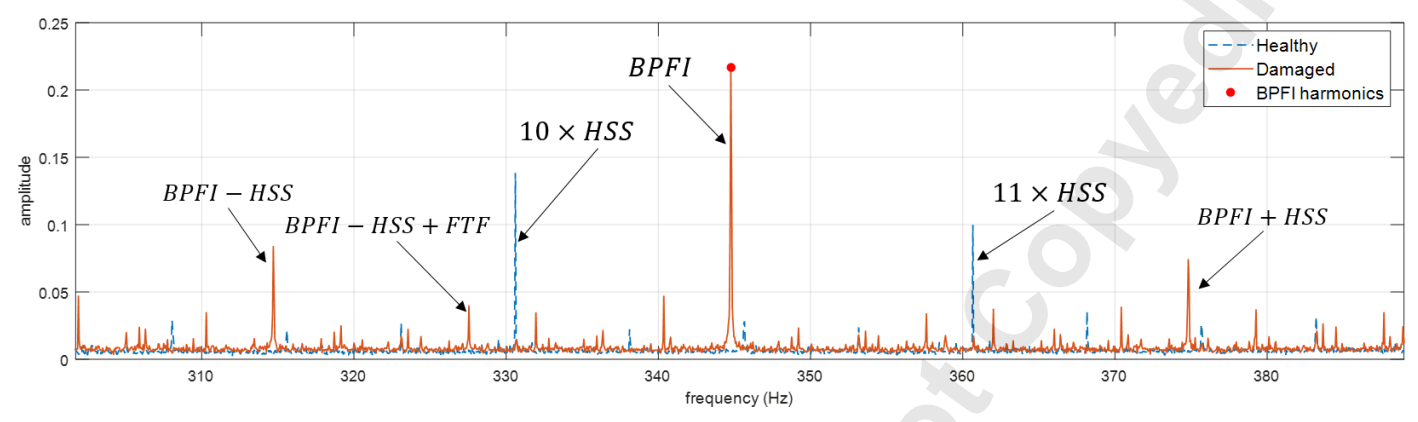

Fig. 7: ZOOM OF AN7 SENSOR DATA EESFO AROUND THE BPFI.

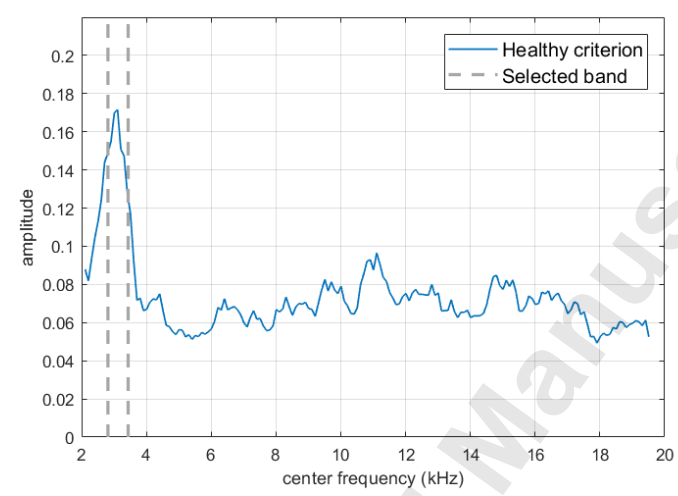

(a)

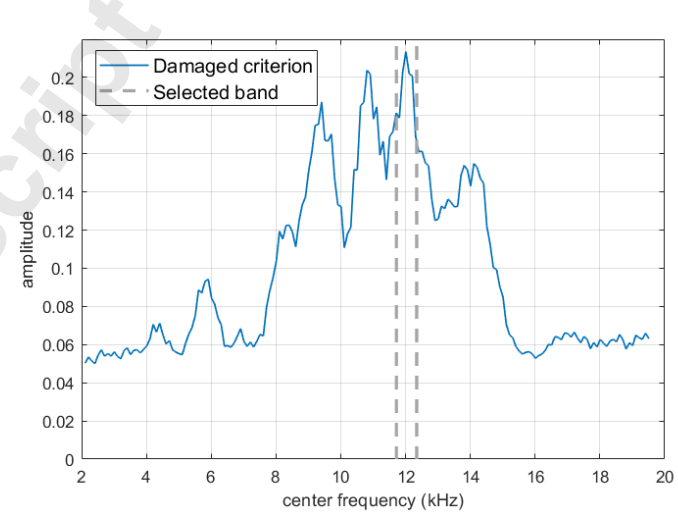

(b)

Fig. 8: EESFO CRITERION FOR THE SELECTION Of BAND OF INTEGRATION OF CSC FOR IMS DOWNWIND BEARING BPFO (105 HZ) OPTIMIZATION FOR SENSOR AN6 FOR (A) HEALTHY AND (B) DAMAGED.

Furthermore the signals captured by the sensor A6, which monitors the Intermediate Speed Shaft (IMS) upwind and downwind bearings, are analysed. The methodology is applied sequentially using all the bearing characteristic frequencies, for the healthy and the damaged case. In the case of BPFO indicator (nominal BPFO $=105 \mathrm{~Hz}$ ), a frequency band located at the high frequencies is selected for the damaged case, while in the healthy case a lower frequency band is selected, as shown in Fig. 8. The corresponding envelope spectrum of the healthy signal presents a number of shaft and gear harmonics, while in the damaged one the harmonics of the BPFO are clearly identified. A zoom on the three harmonics of the BPFO can be found in Fig. 9. The $2^{\text {nd }}$ harmonic of the BPFO coincides with the $7^{\text {th }}$ harmonic of the HSS and the distinction of the peaks between healthy and damaged case is not clear. On the other hand the detection is clear when analysing the first and the third harmonic of the BPFO, as sidebands at the IMS at $7.6 \mathrm{~Hz}$ are present and the peaks are clearly separated from the discrete frequency peaks of the healthy spectrum. The results are validated by the NREL report where damage on the outer race is reported on the downwind bearings. Furthermore using the BPFI indicator, the inner race damage reported on the upwind bearings of the same IMS can be detected. The nominal BPFI for the upwind bearings is equal to $73.7 \mathrm{~Hz}$. The application 
Copyright of the EESFO leads again to the extraction of the Enhanced Envelope Spectrum, where the first and the third harmonics of the BPFI are clearly detected, as presented respectively in Fig. 10a and Fig. 10b.

Finally the approach is applied on the signals captured by the sensor AN5 which monitors the Planetary Carrier (PLC) upwind bearings. The damage report [12] stated that the bearing presented a fretting corrosion. The partners who participated at the NREL wind turbine round robin failed to detect this fault [12] in both the "blind" and "post analysis" round, probably because it occurred at a very low frequency with a very low amplitude, therefore it is masked by the level of noise. The classical Envelope Analysis has been applied on the signal but the characteristic fault frequencies has not been identified at the corresponding spectrum. Moreover the well known Kurtogram has been applied in order to select the "optimum" bandpass filter. The Kurtogram selected a band which finally identified the HSS and IMS gear set fault. The corresponding envelop spectrum after the automated filter band selection by the Kurtogram is presented in Fig. 11. The fault frequency cannot be identified at the spectrum but peaks at the IMS and HSS shaft frequencies that modulate the gear meshing of the damaged gears can can be detected. However the EESFO method applied using as a cyclic frequency the corresponding BPFO, equal nominally to $8.8 \mathrm{~Hz}$, detect clearly the defect. The enhanced envelope spectrum for the healthy and the damaged is presented in Fig. 12. Similar to the other cases, the gear and shaft frequencies are present in the healthy case, while the harmonics of the BPFO are clearly identified in the damaged case. The BPFO is also close to the $4^{\text {th }}$ harmonic of the Sun Shaft frequency at $8.4 \mathrm{~Hz}$. However, thanks to the high resolution of the method, the $4^{\text {th }}$ harmonic of the Sun Shaft appears as a clear sharp peak in the damaged case next to the BPFO. Based on the aforementioned results, it can be concluded that the CSC based methods present higher diagnostic effectiveness compared to power diagnostic tools such as the combination of envelope analysis with the kurtogram. At the time being, the software script of the methodology has not been optimised. On the other hand it should be noted that the EESFO script needs almost 5 minutes to process two signals of 20 seconds each which is of course, longer compared to the 10 seconds which are needed by the envelope analysis combined with the kurtogram.

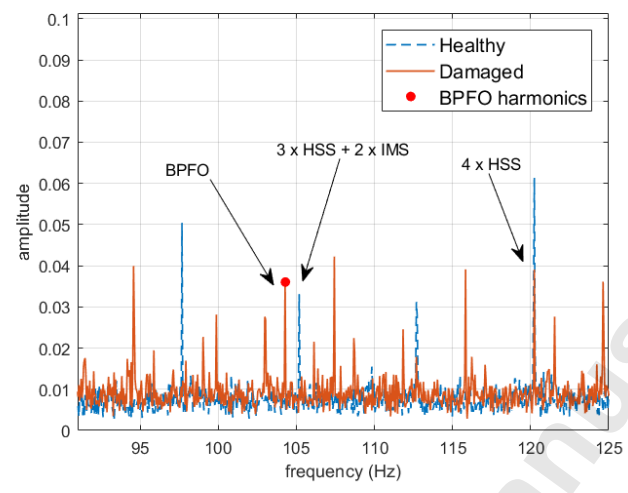

(a)

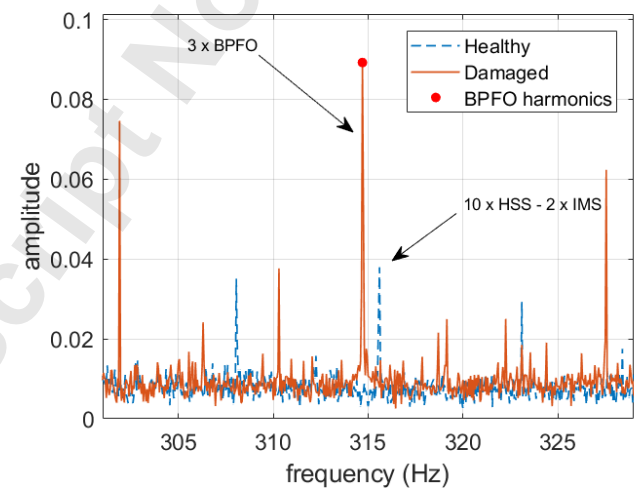

(b)

Fig. 9: EESFO FOR THE BPFO DETECTION OF THE IMS DOWNWIND BEARING DAMAGE CORRESPONDING TO SENSOR AN6 ZOOMED AROUND (A) THE BPFO, (B) $3^{\text {rd }}$ BPFO.

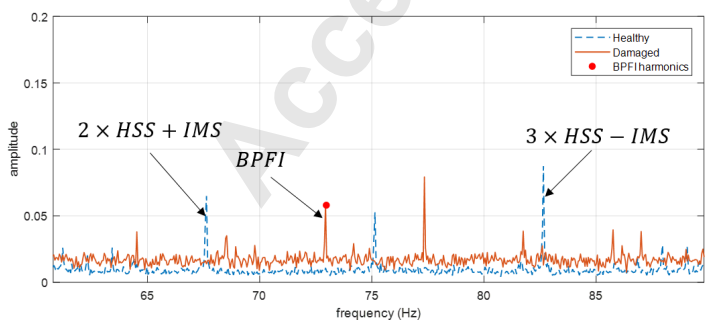

(a)

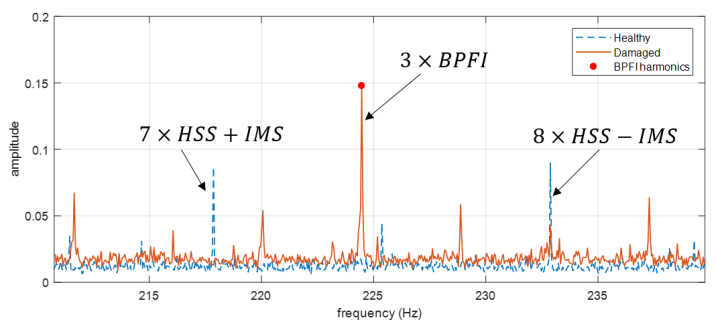

(b)

Fig. 10: ZOOM OF THE EESFO AROUND THE (A) BPFI OF THE IMS UPWIND BEARING. (B) $3^{\text {rd }}$ HARMONIC OF THE BPFI OF THE IMS UPWIND BEARING.

GTP-18-1329 - Gryllias - Page 9 


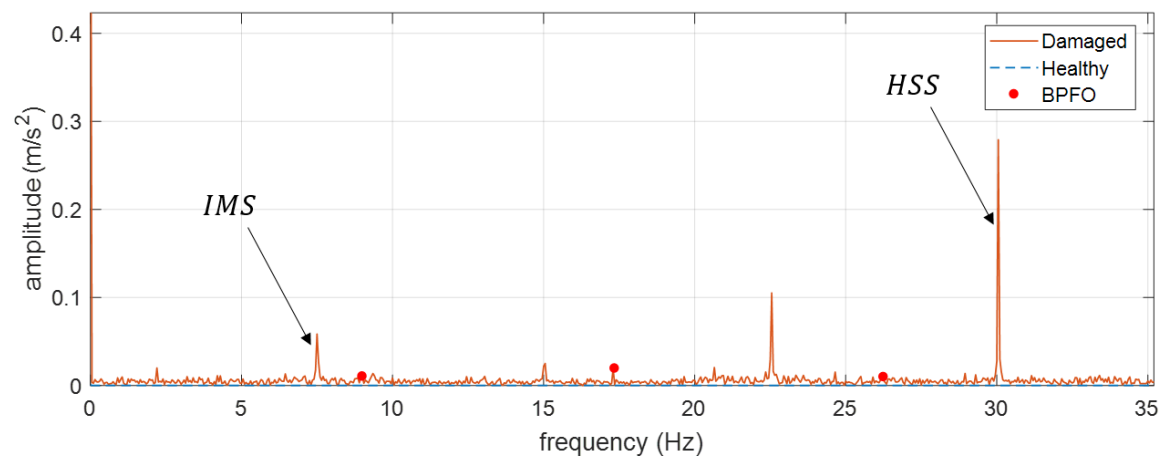

Fig. 11: ENVELOPE SPECTRUM WITH KURTOGRAM FOR AN5.

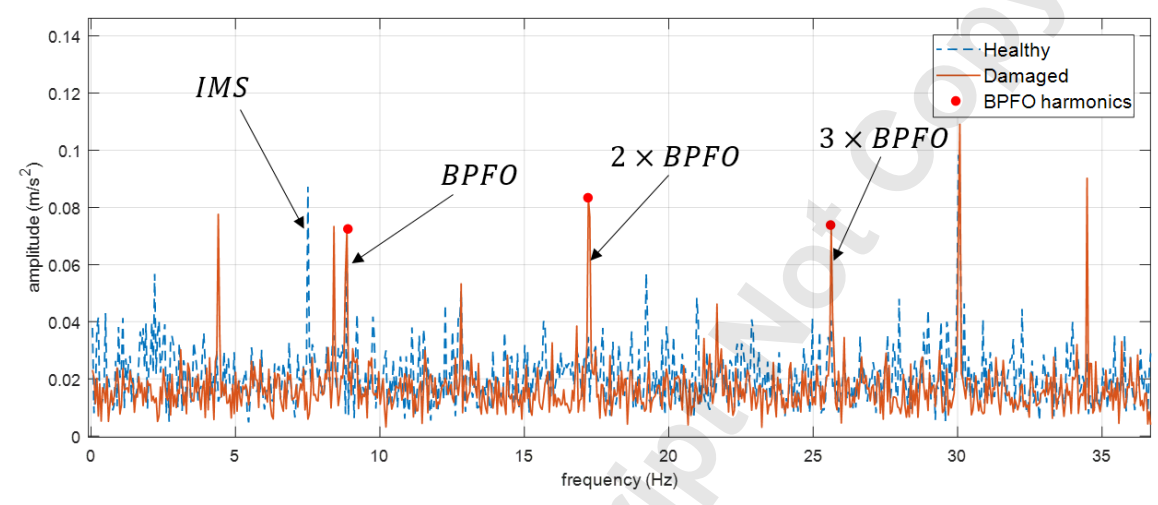

Fig. 12: EESFO FOR THE BPFO DETECTION OF THE PLC UPWIND BEARING DAMAGE CORRESPONDING TO SENSOR AN5.

\section{CONCLUSIONS}

A novel approach for bearing diagnostics has been proposed in this paper. The methodology is based on the Cyclic Spectral Coherence and an automated optimisation procedure has been developed in order to detect the optimal integration band of the CSC map, leading to the Enhanced Envelope Spectrum by Feature Optimization (EESFO). The methodology is tested and validated on a wind turbine gearbox dataset provided by NREL. The proposed methodology successfully detects all the bearing defects generated on the wind turbine gearbox. It should be highlighted that one of the defects has not been detected by the state of the art methodologies as it has been mentioned in the initial report of NREL and was validated by the authors using the envelope anaysis supported by the kurtogram. As a result it can be concluded that EESFO is a promising signal processing tool useful for condition monitoring of complex systems. As future work, the authors are currently working towards the extension of the methodology in order to cover the time varying operating conditions of wind turbines and other complicated mechatronic systems.

\section{Acknowledgements}

The authors would like to gratefully acknowledge Flanders Make and VLAIO Flanders Innovation and Entrepreneurship Agency within the VIBMON - Cost-effective vibroacousic monitoring project for their support. K. Gryllias would like to gratefully acknowledge the Research Fund KU Leuven.The authors would like to thank the National Renewable Energy Laboratory for organizing the test on condition monitoring of the wind turbine gearbox and providing the resulting datasets. The National Renewable Energy Laboratory is funded by U.S. Department of Energy under contract No.DEAC36-08GO28308. 


\section{Copyright Reíerences}

[1] Musial, W., Beiter, P., Schwabe, P., Tian, T., Stehly, T., Spitsen, P., Robertson, A., and Gevorgian, V., 2017. “2016 offshore wind technologies market report". NREL/TP-5000-68587; DOE/GO-102017-5031.

[2] Bawden, T., 2015. Wind power now uk's cheapest source of electricity but the government continues to resist onshore turbines. http://www.independent.co.uk/environment/wind-power-now-the-cheapest-source-of-electricity-butthe-government-continues-to-resist-onshore-a6685326.html, Oct.

[3] Scholbrock, A., Fleming, P., Wright, A., Wang, N., Schlipf, D., and Johnson, K., 2016. "Lidar-enhanced wind turbine control: Past, present, and future". In Proceedings of the 2016 American Control Conference Boston, Massachusetts, Academic Press, pp. 1-8.

[4] Lebold, M., McClintic, K., Campbell, R., Byington, C., and Maynard, K., 2000. "Review of vibration analysis methods for gearbox diagnostics and prognostics". In Proceedings of the 54th Meeting of the Society for Machinery Failure Prevention Technology, Academic Press, pp. 623-634.

[5] Antoni, J., and Randall, R. B., 2006. "The spectral kurtosis: application to the vibratory surveillance and diagnostics of rotating machines". Mechanical Systems and Signal Processing, 20(2), pp. 308 - 331.

[6] Gryllias, K., and Antoniadis, I., 2009. "A peak energy criterion (p.e.) for the selection of resonance bands in complex shifted morlet wavelet (csmw) based demodltaion of defective rolling element bearngs vibration response". International Journal of Wavelets, Multiresolution and Information Processing, 7(4).

[7] Randall, R. B., and Sawalhi, N., 2011. "Use of the cepstrum to remove selected discrete frequency components from a time signal". Springer New York, New York, NY.

[8] Borghesani, P., Pennacchi, P., Randall, R., Sawalhi, N., and Ricci, R., 2013. "Application of cepstrum pre-whitening for the diagnosis of bearing faults under variable speed conditions". Mechanical Systems and Signal Processing, 36(2), pp. $370-384$.

[9] Antoni, J., 2007. “Cyclic spectral analysis in practice”. Mechanical Systems and Signal Processing, 21(2), pp. 597 630.

[10] Antoni, J., Xin, G., and Hamzaoui, N., 2017. "Fast computation of the spectral correlation". Mechanical Systems and Signal Processing, 92, pp. $248-277$.

[11] Sheng, S., 2012. "Wind turbine gearbox vibration condition monitoring benchmarking datasets". NREL/TP-500054530 .

[12] Sheng, S., 2012. "Wind turbine gearbox condition monitoring round robin study vibration analysis". NREL/TP-500054530 .

[13] Sheldon, J., Mott, G., Lee, H., and Watson, M. "Robust wind turbine gearbox fault detection". Wind Energy, 17(5). 